\title{
GESTÃO POR PROCESSOS E A GESTÃO DE PROJETOS: UM MODELO GERENCIAL PARA ALOCAÇÃO DE RECURSOS
}

\section{PROCESS AND PROJECT MANAGEMENT: A MANAGEMENT MODEL FOR RESOURCE ALLOCATION}

\author{
Marco Aurélio Moroni (UFRGS) ${ }^{1}$ Peter Bent Hansen (PUCRS) ${ }^{2}$ \\ ${ }^{1}$ VEM (Varig Engenharia e Manutenção) \\ marco.moroni@brturbo.com.br \\ ${ }^{2}$ Prof. Adjunto Administração e Eng. De Produção - PUC RS \\ peter.hansen@pucrs.br
}

\begin{abstract}
Resumo
Nos processos de negócios dos dias atuais, a antiga visão funcional tem dado lugar à visão porprocessos. Nesta abordagem, diversos departamentos de uma organização executam etapas distintas de processos corporativos que cruzam a organização horizontalmente. Além dos processos de negócios, algumas organizações apresentam necessidade de implantação de projetos específicos. No entanto, a gestão por processos e de projetos pode demandar recursos de um mesmo departamento, exigindo planejamento e controle adequados. Este artigo traz a discussão de uma proposta de integração da gestão por processos e da gestão de projetos, propondo um modelode gestão que permita a utilização das duas metodologias, visando otimização dos recursos da empresa.
\end{abstract}

Palavras Chave: Manutenção Aeronáutica, Gestão por Processos, Gestão de Projeto, Integração.

\section{Introdução}

Durante o processo de reavaliação estratégica, uma empresa de manutenção aeronáutica, que aqui denominaremos de AVIAMAN, decidiu utilizar o modelo BSC - Balanced Scorecard para mapear e medir o desempenho dos objetivos estabelecidos no seu planejamento estratégico.

Durante o programa de implantação do $\mathrm{BSC}^{1}$, surgiram algumas iniciativas que estavam diretamente associadas aos processos existentes, mapeados quando da implantação de um sistema de gestão ERP $^{2}$. Outras iniciativas tinham a característica de projetos, e para conduzir tais projetos a empresa optou por adotar a metodologia proposta pelo PMI - Project Management Institute.

Como se tratam de processos e projetos de caráter essencialmente técnico e que exigiriam domínio tecnológico associado a diferentes departamentos, tanto os processos como os projetos deveriam ser conduzidos através do compartilhamento de recursos destes mesmos departamentos. 
O grande desafio então se concentrou em entender como poderiam os recursos dos diversos departamentos serem compartilhados de modo a atender as demandas dos processos e dos projetos ao mesmo tempo. Este artigo traz à discussão este cenário, buscando identificar e propor alternativas para as dificuldades e limitações impostas quando da aplicação de ambos os modelos de gestão concomitantemente.

\section{A Gestão por Processos}

Na prestação de serviços, os processos estão intimamente relacionados com o desempenho final, isto é, o cliente percebe o serviço como um todo, e não suas partes de forma separada. As estruturas funcionais apresentam deficiências no fluxo de atividades entre os departamentos, ocasionando diversos problemas para a entrega do produto final. Estimativas indicam uma proporção de problemas em processos de $94 \%$ contra $6 \%$ oriundos de outras causas (Deming apud Harrington, 1993).

\subsection{A Visão Funcional Tradicional}

Estruturas verticalizadas, nas quais a administração é conduzida setorialmente ou por função, apresentam problemas para os administradores, pois não criam um fluxo cadenciado desde a criação até a entrega dos serviços. A troca da visão da organização funcional (setorial) para a visão por processos tem sido apontada como o caminho para integração entre os departamentos (Orssatto; 1999). Isto só pode ser obtido se a organização passar a concentrar sua atenção nos processos que controlam as interações com os clientes (Harrington, 1993).

As estruturas tradicionais, construídas sobre um modelo baseado em departamentos praticamente independentes, parecem não mais atender às solicitações atuais dos mercados e sua dinâmica. Tipicamente, as representações das estruturas tradicionais não mostram aspectos relacionados com clientes, fornecedores ou relacionamentos interdepartamentais, e se apresentam como um conjunto de funções desconexas, ocasionadas pela forma departamental de organizar as empresas (Orssatto, 1999).

Modelos voltados aos processos empresariais estão sendo adotados na tentativa de atender às demandas que o mercado está impondo às organizações. Embora sua implementação geralmente não seja uma tarefa fácil, já existem organizações implantando tais modelos, pois esta mudança pode criar significativa vantagem competitiva para a organização. 


\subsection{Análise de Processos, uma Visão Horizontal}

A visão tradicional (funcional) das organizações produz lacunas interdepartamentais e dificulta a obtenção de uma visão corporativa. A proposição de uma gestão por processos, proporciona uma visão mais sistematizada da organização e seus departamentos, identificando as deficiências interdepartamentais que por ventura existirem.

A utilização da gestão por processos combinada com uma gestão vertical provoca o surgimento de várias lacunas e sobreposições podendo gerar ineficiência (Harrington, 1993). As lacunas geradas entre departamentos são consideradas as melhores oportunidades para investimento em melhorias e obtenção de resultados (Rummler e Brache, 1994). Assim, as organizações podem ser consideradas como sistemas que processam diversas entradas e geram saídas, através de diversos processos que cruzam a organização horizontalmente (Hronec, 1993; Campos, 1998; Enström, 2002).

\subsection{Os Níveis da Organização sob a Ótica de Processos}

Ao ser considerada como um sistema, a empresa pode ser analisada desde seu nível de negócios até os níveis operacionais. Rummler e Brache (1994) propõem três níveis para identificação dos processos/atividades - Nivel da Organização, Nível de Processos e Nível de Trabalho/Executor. No primeiro nível Rummler e Brache (1994) apresentam o modelo de Mapa de Relacionamentos, onde os processos e suas ligações dentro e fora da empresa são identificados. Neste mapa pode-se identificar os macro processos e como eles cruzam a organização em sua estrutura hierárquica. No nível de processo os macro processos são detalhados em nível departamental utilizando basicamente três variáveis: objetivos do processo, o projeto do processo e o gerenciamento do processo. Rummler e Brache (1994), propõem que os objetivos dos processos externos devem ser movidos por necessidades explícitas de clientes externos, e os objetivos internos devem estar em sintonia com as necessidades destes e dos clientes internos. Rummler e Brache, (1994), Hronec (1993), Slack et al. (2002), Harrington (1993), lançam mão de fluxogramas interdepartamentais para identificação das áreas envolvidas em cada processo. No último nível, do trabalho/executor, Rummler e Brache (1994) ressaltam a importância dos funcionários na execução dos processos. De nada serve um processo com objetivos claros e um projeto bem definido se o elemento humano envolvido não está treinado ou preparado para fazer com que o processo seja executado eficientemente.

\section{Gestão de Projetos}

Os projetos sempre estiveram presentes nas organizações de forma sistematizada ou não. Mais recentemente diversas iniciativas vem sendo adotadas para estabelecer padrões de 
gerenciamento de projetos, que possam ser adotados nos mais variados segmentos da indústria. $\mathrm{O}$ Project Management Institute - PMI vem consolidando as melhores práticas no gerenciamento de projetos e publicando-as em seu PMBOK - Project Management Body of Knowledge.

Segundo (PMBOK, 2000; Dinsmore, Cavalieri, 2003) o ciclo básico do gerenciamento de projetos pode ser dividido em cinco etapas, como apresentado na Figura 1, que compõem diversos processos: (i) processos de iniciação; (ii) processos de planejamento; (iii) processos de controle; (iv) processos de execução; (v) processos de encerramento. Na fase de iniciação são estabelecidas as apresentações do projeto, escopo, autorizações, etc. Na fase de planejamento o projeto é refinado, as atividades são definidas, são alocados os recursos, são estimados os custos e as opções para atingir os objetivos são determinadas. Na fase execução e controle se estabelecem os princípios para o controle de pessoas e recursos e monitoramento do andamento das etapas do projeto. A última etapa diz respeito ao encerramento do projeto; documentos de encerramento são criados e relatórios são produzidos para registrar os pontos positivos e negativos do projeto.

De forma semelhante à gestão por processos a gestão de projetos também propõe uma visão horizontalizada da organização fazendo uso dos recursos da empresa, alocando-os temporariamente para a produção de um bem ou serviço (Muñoz-Avila et al., 2001). Na visão funcional, cada funcionário/departamento possui uma hierarquia bem definida, os membros da equipe de projetos são agrupados por especialidade e o escopo dos projetos em tais organizações é limitado às fronteiras funcionais. Cada departamento trabalha ou entende que seu trabalho está concluído quando sua etapa assim o estiver. O gerente de projetos busca aproveitar ao máximo os recursos da organização, enquanto o gerente de função enfatiza a alocação de horas de seus subordinados no departamento (Lavine, 2003).

Figura 1: Ciclo de controle de projetos

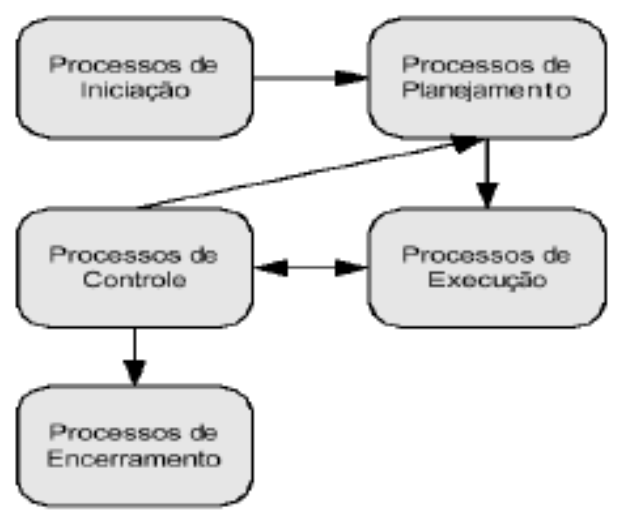

Fonte: PMBOK (2000, p.31), DINSMORE e CAVALIERI (2003, p.5) 
A Figura 2 (a) e (b) apresentam os extremos propostos no PMBOK em termos organizacionais, incluindo a estrutura funcional tradicional e a estrutura projetizada proposta pelo PMI. Entre estes extremos o PMI também propõe diversas estruturas organizacionais intermediárias.

Em todos os modelos apresentados pelo PMI, sugere-se uma estrutura matricial, importante para a otimização da utilização de recursos. As organizações devem utilizar os conceitos de uma organização matricial para obter as vantagens deste modelo; basta que se estabeleçam as regras para que os recursos sejam cedidos ao projeto (Toledo, 2003).

Figura 2: Visão Funcional (a) e Visão Projetizada (b)

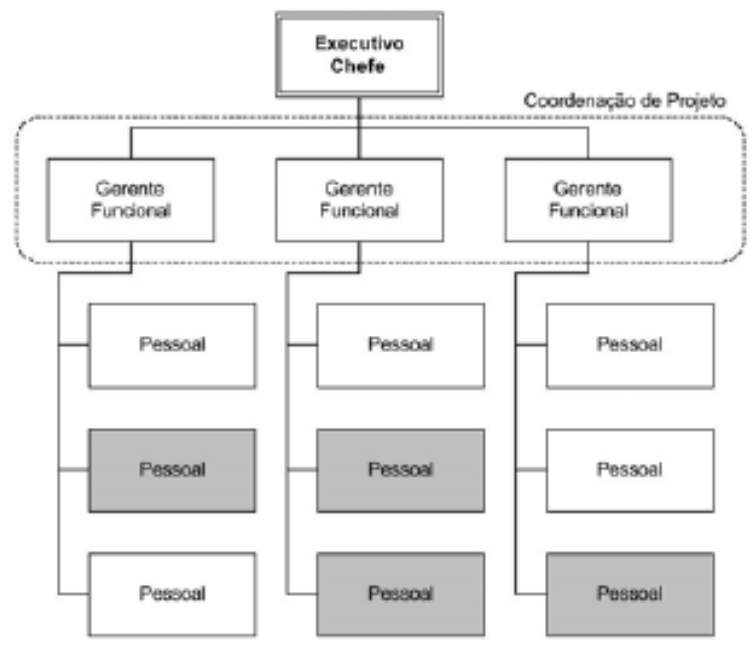

(a)

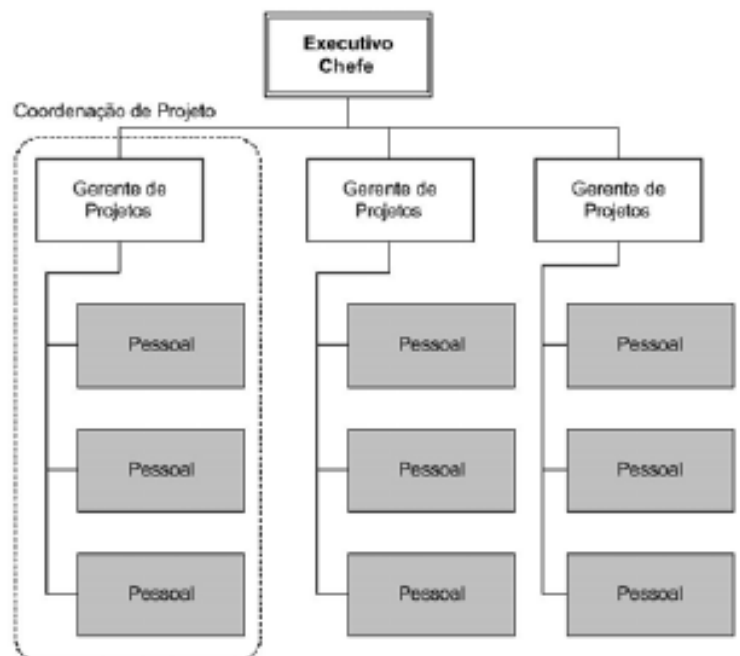

(b)

Fonte: PMBOK (2000, p.20-21)

\section{Gestão por Processos e Gerenciamento de Projetos: Considerações sobre sua Implementação}

Em uma atividade que precedeu a implantação do sistema ERP, a empresa em estudo redesenhou seus processos. Esta atividade partiu dos macro-processos e seguiu o detalhamento até que as atividades pudessem ser implementadas de forma otimizada no sistema. Ainda em uma fase anterior ao projeto do sistema de informações, durante o mapeamento dos objetivos estratégicos em um BSC, a empresa já havia desenvolvido algumas iniciativas que tinham uma visão orientada aos processos. Em alguns casos eram estabelecidos gerentes responsáveis pelas diversas etapas do processo em todo o ciclo dentro da organização. Ao mesmo tempo em que tinham esta delegação, também respondiam para o gerente funcional pelas atividades de rotina inerentes ao departamento. Além dos processos já estabelecidos, a organização identificou diversas atividades com perfil de projeto, i.e., utilizam temporariamente recursos de diversas especialidades e possuem um ciclo 
finito.

Neste momento buscaram-se identificar metodologias de gerenciamento de projetos que pudessem ser ajustadas às necessidades da empresa de forma a manter os processos já estabelecidos. Deveria haver um meio de conciliar a gestão por processos e a gestão de projetos de modo harmonizado fazendo uso dos mesmos recursos.

\section{Necessidades e Requisitos do Modelo}

Manter as metodologias de processos e de projetos tornou-se então uma premissa, e diversas dúvidas quanto à sua aplicação surgiram. Como visto anteriormente, tanto o gerenciamento por processos como o gerenciamento de projetos estabelece rotinas que seguem horizontalmente a estrutura da organização. Um gerente de processo/projeto é designado como responsável por todo o ciclo dentro da organização, independentemente do departamento a que pertence. Os indivíduos alocados para realizar os processos ou para participar de projetos possuem características específicas e podem ser oriundos de diversas áreas da empresa. Esta similaridade despertou a atenção do grupo gerencial que decidiu então avaliar a possibilidade de se desenvolver um modelo que pudesse conciliar ambas as metodologias.

Após a avaliação dos modelos apresentados na literatura pesquisada foi possível estabelecer algumas recomendações, que foram levadas em consideração para a criação do modelo em questão: (i) proporcionar continuidade aos processos já estabelecidos na organização; (ii) permitir compartilhamento de recursos entre a gestão dos processos estabelecidos e o gerenciamento de projetos; (iii) permitir acompanhamento do desempenho dos processos e da evolução dos projetos; (iv) estabelecer um meio de priorizar os processos de modo a permitir a alocação de recursos em projetos; (v) identificar as funções afetadas pelos processos priorizados, de modo a permitir ao gerente a utilização de critérios na alocação de recursos aos projetos; (vi) estabelecer uma rotina de negociação entre o gerenciamento de projetos e o gerenciamento por processos; (vii) estabelecer um meio de avaliação do desempenho dos projetos.

\section{Proposição de um Modelo}

O modelo resultante da pesquisa realizada pelo grupo gerencial apresenta uma utilização complementar de metodologias previstas no gerenciamento por processos e o gerenciamento de projetos proposto pelo PMI. O modelo propõe uma visão organizacional definindo como os recursos serão alocados e uma visão de fluxo de operacional que estabelece as interações consideradas necessárias entre a gestão por processos e a gestão de projetos, para que o resultado 
final satisfaça as necessidades da empresa.

\subsection{Visão Organizacional}

Sob a ótica organizacional o modelo propõe a utilização de uma estrutura matricial onde o gerente funcional dá lugar ao gerente de operações, responsável pela alocação de recursos aos processos e aos projetos. Os gerentes de processos estarão inseridos na organização não necessariamente em posições hierárquicas, mas serão responsáveis por seus processos desde seu inicio até a entrega do produto final. A alocação destes recursos será de responsabilidade do gerente de operações e se dará pela priorização dos processos.

Figura 3: Modelo Gerencial Organizacional Proposto

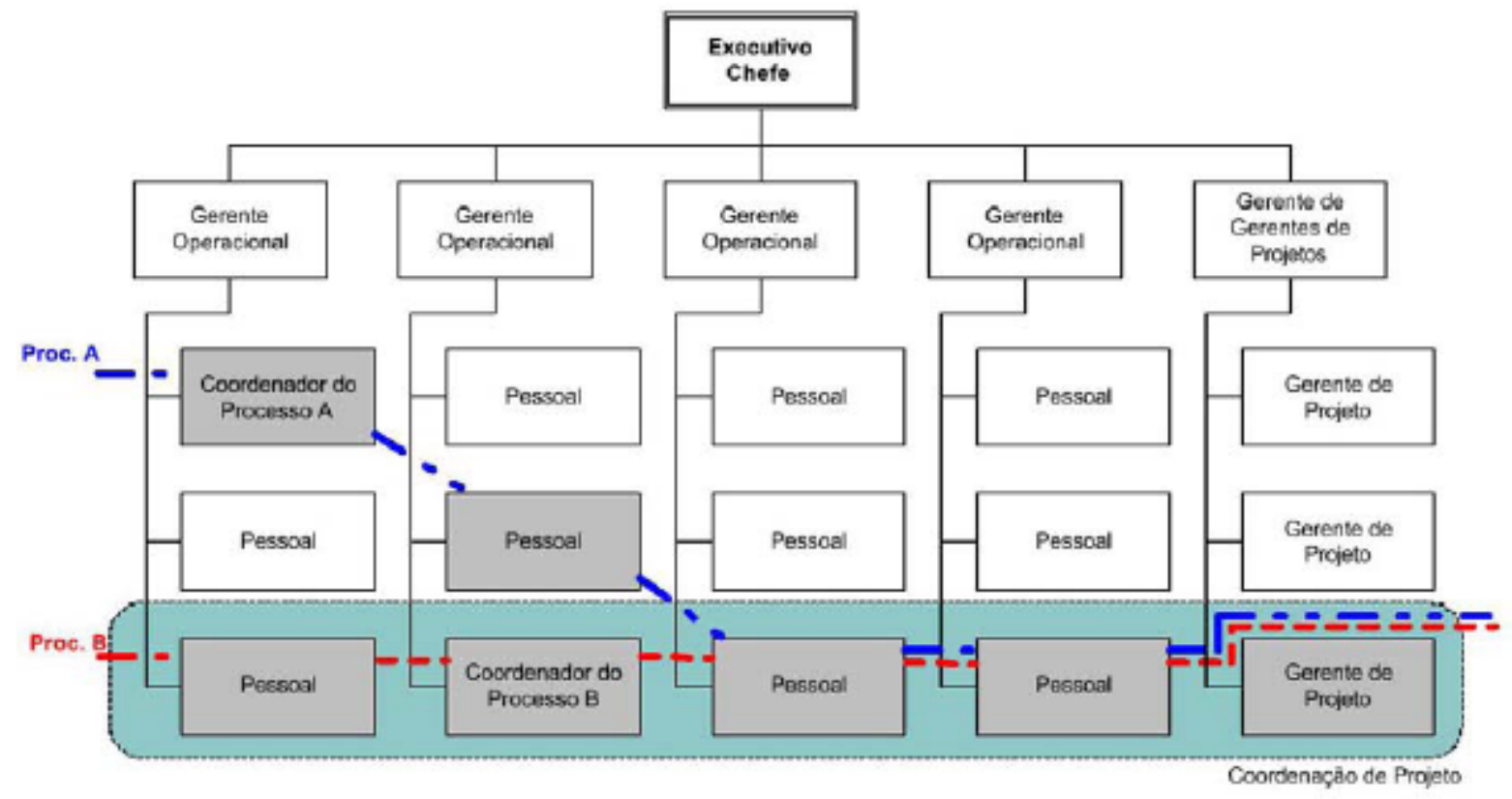

Quanto à visão de projetos, a organização proposta tem como origem uma das matrizes propostas pelo PMI. Um gerente de projetos será alocado a cada projeto e será responsável por sua execução desde seu início até sua finalização, em todas as etapas dentro dos diversos departamentos. A alocação de recursos será negociada com o gerente de operações. Baseando-se nas informações de priorização dos processos e na utilização dos recursos para as funções departamentais o gerente operacional irá alocar os recursos compartilhados ao projeto. A Figura 3 apresenta o modelo organizacional proposto pelo modelo, aqui coordenadores de processos gerentes de projetos e a estrutura funcional se mesclam buscando utilizar os recursos disponíveis na organização.

\subsection{Fluxo Operacional}

Do ponto de vista do fluxo operacional o modelo proposto parte do ciclo de gerenciamento de projetos proposto pelo PMI, e da priorização de processos proposta por Moroni, 2003; Moroni e Hansen (2004). A priorização dos processos é baseada nos objetivos estratégicos e na estrutura 
funcional vigente na organização. Entre o ciclo de gerenciamento de projetos e a gestão dos processos, o modelo prevê uma interface que representa a dinâmica de alocação de recursos e de controle de andamento dos projetos, suportada por um sistema de TI que irá conter os dados necessários para dar suporte ao processo decisório.

O ciclo de gerenciamento de projetos proposto pelo PMI é composto pelas cinco etapas indicadas na Figura 1. Na fase de planejamento do projeto, os gerentes de projetos deverão negociar alocação de recursos com os departamentos necessários. O gerente operacional irá negociar a disponibilidade de recursos com base na priorização estabelecida para os processos da empresa. $\mathrm{Na}$ etapa de execução do projeto, os gerentes de projetos irão avaliar o andamento dos projetos através de indicadores associados aos departamentos e recursos alocados. Da mesma forma os gerentes de processos irão avaliar o desempenho dos processos através de indicadores associados aos departamentos envolvidos.

Na Figura 4 pode ser vista uma representação gráfica deste modelo com seus quatro elementos básicos, a gestão de projetos, a gestão por processos, identificação dos inputs e a interface que busca estabelecer procedimentos para a alocação de recursos necessários às duas metodologias.

Figura 4: Fluxo de Gerenciamento de Projetos/Processos Proposto

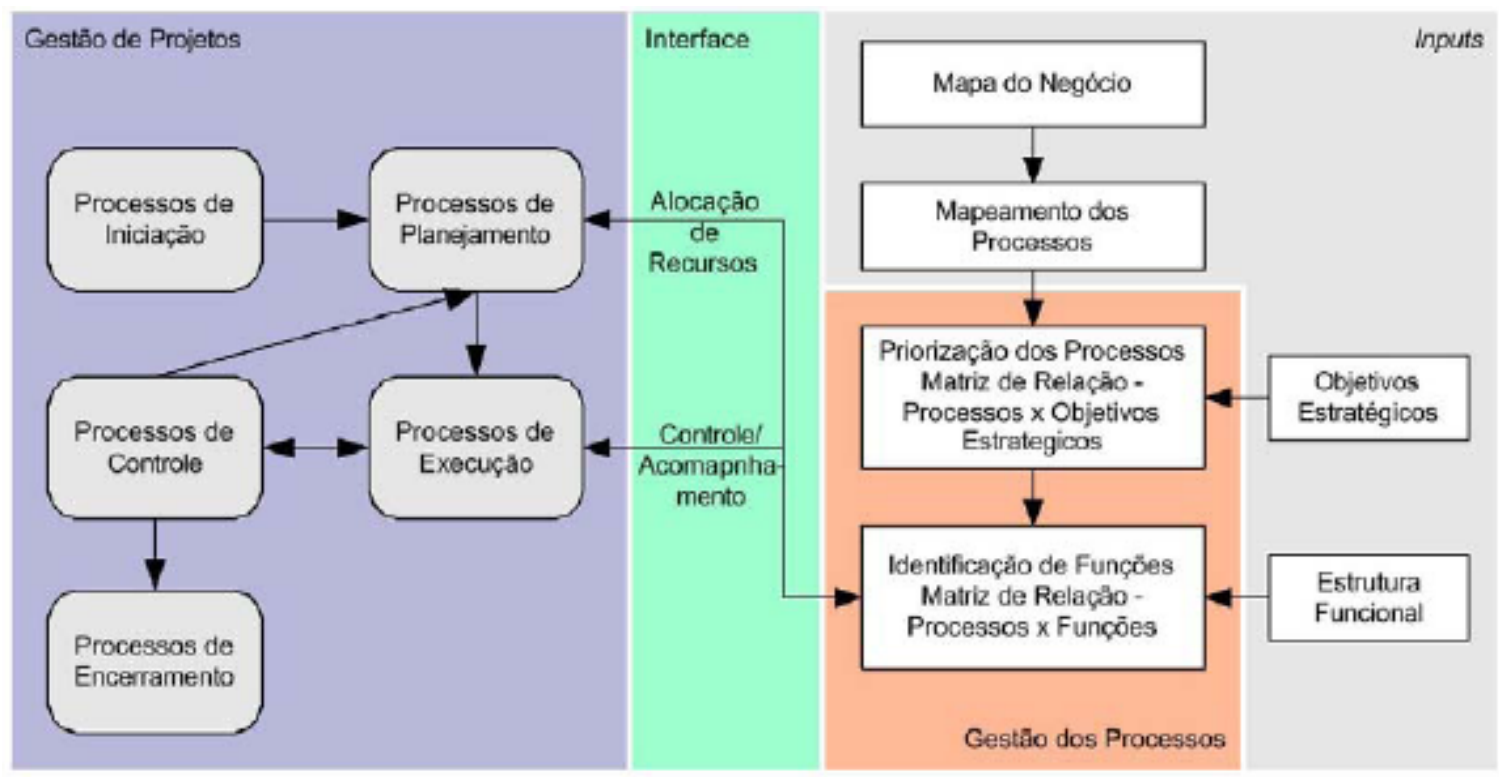

Os Inputs compreendem quatro etapas iniciais. Nestas, são produzidas informações necessárias para a priorização dos processos e para a identificação das funções (departamentos) envolvidas nos processos prioritários.

A primeira etapa compreende a elaboração de um mapa de relacionamentos que apresente a 
empresa e suas relações externas como, por exemplo, com fornecedores e clientes. O modelo proposto por este trabalho lança mão da metodologia adaptada das propostas de Rummler e Brache (1994) e Hronec (1993). Na segunda etapa os processos deverão ser identificados partindo-se das definições do mapa de relacionamentos criado para a empresa na etapa inicial do modelo. Esta descrição visa criar conhecimento detalhado da operação da organização e definição dos indicadores a serem utilizados no sistema de avaliação de desempenho incluído no modelo proposto. O terceiro e quarto Inputs referem-se aos objetivos estratégicos e à estrutura funcional da empresa.

\subsection{Alocação dos Recursos e Acompanhamento de Projetos / Processos}

A interface entre os modelos de gestão de projetos e de processos estabelece uma rotina para a alocação e controle de utilização de recursos de uma forma sistematizada. Na alocação o gestor de projetos identifica os recursos necessários e existentes na organização considerando sua disponibilidade.

Por definição a organização toma como ponto de partida os processos estabelecidos, i.é., a alocação de recursos é primeiramente designada pelos gerentes operacionais para atender os processos estabelecidos, priorizados e sob a tutela dos coordenadores de processos.

A utilização dos recursos nos processos cria limitações que serão impostas aos gestores de projetos na alocação destes mesmos recursos. Na fase de planejamento, o gerente de projetos deve considerar tais limitações eventualmente estendendo prazos se necessário.

No caso de mão-de-obra, por exemplo, o gestor de projetos deve considerar esta disponibilidade na construção do plano de projetos. Para os casos onde não houver disponibilidade de certo recurso, o gestor de projetos poderá considerar a contratação externa.

Os gerentes operacionais monitoram a utilização de seus recursos através de métricas relacionadas ao tipo de recurso que estão cedendo aos processos/projetos. No caso de mão-de-obra serão controlados as $\mathrm{h} / \mathrm{h}^{3}$ alocados, da mesma forma recursos como energia elétrica, água e outros podem ter seus controle via unidades de consumo ( $\mathrm{Kw}$, litros, etc.).

$\mathrm{Na}$ etapa de Controle/Acompanhamento, o gestor de projetos avalia quanto do recurso planejado é de fato utilizado na execução do projeto através da análise de valor agregado (PMBOK, 2000; Vargas, 2003). Sob a ótica dos processos, sua avaliação se dá através da análise dos índices de desempenho estabelecidos para o processo. É interessante observar que em determinadas situações um departamento/indivíduo poderá estar sendo avaliado sob duas perspectivas, uma relacionada com sua capacidade de agregar valor ao projeto e outra sob a luz dos resultados do processo ao qual ele faz parte.

3 Horas homem, quantidade de horas e de homens alocados a uma atividade específica. 
Dependendo to tipo de atividades que compõem um processo, o departamento/indivíduo estará sendo avaliado pelos resultados obtidos nas atividades chave, ou no processo como um todo.

\title{
7 Considerações Finais
}

Com os processos priorizados e seu impacto identificado nas funções da empresa, entendese que o modelo proporciona informações para que os gerentes operacionais (funções) possam dimensionar as alocações de recursos racionalmente.

O modelo mantém o ciclo de gerenciamento de projetos propondo uma interface com a gestão de processos. Esta interface visa estabelecer uma rotina sistematizada para alocação e dimensionamento de recursos de forma a manter o desempenho dos processos em níveis aceitáveis e, ao mesmo tempo, alocar recursos para projetos específicos.

Por conveniência, e aproveitamento de recursos, o modelo proposto neste artigo fez uso do sistema de gerenciamento de processos já desenvolvido durante o programa de implantação do BSC na organização pesquisada.

As atividades desenvolvidas pela empresa em estudo neste trabalho possuem um caráter fundamentalmente técnico e com nível de especialização, que torna necessária uma utilização dos recursos de forma mais otimizada possível. Isto implica um balanceamento na alocação de recursos entre os processos e os projetos, função para a qual, se acredita, o modelo proposto será de grande contribuição.

Como a organização está em fase de refinamento do processo estratégico através da utilização do BSC, a aplicação do modelo será avaliada quando a implantação das ações definidas a partir dos objetivos estratégicos estiver concluída.

Atualmente investiga-se na organização o mapeamento do modelo e seus requisitos em um sistema de informações com base na integração de recursos de bancos de dados e de aplicativos de gerenciamento de projetos/processos.

\begin{abstract}
In nowadays business process, the old functional view is being replaced by a process view. In this approach, several departments accomplish different steps of corporate processes that horizontally cross the whole organization. Beyond the business process some organizations need implementation of specific projects. In this scenario business process and project management may share resources from one department demanding management control. An aeronautical maintenance company has its maintenance processes that cross the organization and thus falls in the above category. This paper brings to discussion an integration of process management with project management, and proposes a management model that integrates both methodologies focusing in the company resources sharing and optimization.
\end{abstract}

Key-words: Manutenção Aeronáutica, Gestão por Processos, Gestão de Projeto, Integração. 


\section{Referências}

CAMPOS, Jose A.; Cenário Balanceado - Balanced Scorecard - Painel de Indicadores para a Gestão Estratégica dos Negócios, Editora Aquariana, SP, 1998.

DINSMORE, Paul C., CAVALIERI, Adriane; Como se Tornar um Profissional em Gerenciamento de Projetos, Editora Qualitymark, SP, 2003.

ENSTRÖM, Josefin; Developing Guidelines for Managing Processes by Objectives, Master’s Thesis, Gothemburg, 2002.

HANSEN, Peter B., MORONI, Marco A.; Gestão e Avaliação de Desempenho: Um Modelo Conceitual para uma Empresa de Manutenção Aeronáutica, Anais XXIV Congresso Nacional de Engenharia de Produção, 2004.

HARRINGTON, James H.; $\quad$ Aperfeiçoando Processos Empresarias - Estratégia Revolucionária para o Aperfeiçoamento da Qualidade, da Produtividade e da Competitividade, Editora Makron Books, SP, 1993.

HRONEC, Steven, M.; Vital Signs - Using Qaulity, Time, and Cost Performance Measurements to Chart your Company's Future, Arthur Andersen Co., NY, 1993.

LAVINE, Harvey A.; Practical Project Management: Tips, Tactics, and Tools, AEW Services, Vancouver, BC, 2003.

MORONI, Marco A.; Serviços de Manutenção Aeronáutica como Unidade de Negócios: Um Modelo de Gestão Baseado num Sistema de Indicadores de Desempenho, Dissertação de Mestrado, Universidade Federal do Rio Grande do Sul, Porto Alegre, RS, 2003.

MUÑOZ-AVILA, Hector, GUPTA, Kalyan, AHA, David A., NAU, Dana S.; Knowledge-Based Project Planning, Department of Computer Science, University of Maryland, College Park, MD 20742-3255, 2001.

ORSSATTO, Carlos H.; Busca da Competitividade Através da Gestão por Processos e os Elementos Envolvidos na Mudança, Anais XIX Congresso Nacional de Engenharia de Produção, 1999.

PMBOK Guide; A Guide to the Project Management Body of Knowledge, Project Management Institute, Newtown Square, Pennsylvania, 2000.

RUMMLER, Geary A., BRACHE Alan P.; Melhores Desempenhos das Empresas - Uma abordagem Prática para Transformar as Organizações através da reengenharia, Editora Makron Books, SP, 1994.

SLACK, N., CHAMBERS, S., JOHNSTON, R.; Administração da Produção, Editora Atlas, 2 ed., SP, 2002.

TOLEDO, Nilton N.; O Alinhamento dos Projetos às Estratégias da Organização, Material Didático, PMI São Paulo, 2004.

VARGAS, Ricardo; Valor Agragado em Projetos - Revolucionando o Gerenciamento de Custos e Prazos, Editora Brasport, Rio de Janeiro, RJ, 2003.

\section{Dados completos do primeiro autor:}

Nome completo: Marco Aurelio Moroni

Filiação institucional: VEM (Varig Engenharia e Manutenção)

Função ou cargo ocupado: Gerente de Divisão - Mestrado em Eng. De Produção pela UFRGS

Endereço completo para correspondência: R. Anita Garibaldi 86 apto 402; Centro - Canoas - RS -

Brasil - 92010-100

Telefones para contato: (51) 3358-7186; (51) 3476-5994

e-mail: marco.moroni@brturbo.com.br; marco.moroni@,vem.aero 


\section{Dados completos do Segundo autor}

Nome completo: Peter B. Hansen

Filiação institucional: PUCRS

Função ou cargo ocupado: Prof. Adjunto Administração e Eng. De Produção

Endereço completo para correspondência: Av. Ipiranga 6681, Prédio 50, $11^{\circ}$ Andar, Sala 1105,

Partenon - Porto Alegre - RS - 90619-900

Telefones para contato: (51) 3320-3524

e-mail: peter.hansen@pucrs.br

Recebido para publicação em: 29/11/2005

Aceito para publicação em: 25/01/2006 